\title{
E-learning: Coupling Course Management Systems and Dynamic Taxonomies
}

\author{
Barbara Demo \\ Dipartimento di Informatica \\ Università di Torino - Italy \\ barbara@di.unito.it
}

\author{
Alessio Angius \\ Corso di Studi in Informatica \\ Facoltà di Scienze MFN \\ Università di Torino - Italy \\ angius.alessio@educ.di.unito.it
}

\begin{abstract}
Dynamic taxonomies integrated into e-learning tools play a double role: on the one hand they are a powerful retrieval system in the usually large content base of an e-learning environment, on the other hand they allow and strongly encourage orthogonal visits of available learning resources by exploiting associations the user would not have thought of (and which are the specific contribution of dynamic taxonomies). These two roles are of interest both for teachers, who may use the search engine to retrieve hints for presentations, assignments, etc., and for students, who may explore the whole learning environment in a new profitable way, which makes, for example, immediately available for a same subject different aspects dealt with in different courses. In the paper we describe the integration of dynamic taxonomies into Moodle, a Course Management System for cooperative learning.
\end{abstract}

\section{Introduction}

During the last decade many activities have been undertaken which investigate technology-enhanced learning under different perspectives: from sophisticated virtual laboratories, with games and simulations, to electronic support of distance cooperation for communities [1]. In our department the decision of using a course management system (CMS) was taken in 2003 for two main reasons. On the one hand there was the desire to converge to a more uniform style in the course pages and learning resources: professors and lecturers had been using web publishing from the very beginning of the web and, as a consequence, many different techniques and interface models were present. On the other hand, this was the good occasion for testing and comparing the most popular CMSs in a concrete real-world situation.

A blended-learning approach was decided, where the web technology was aimed to extend teaching and learning outside formal class times. Thus, though not excluding distance learning, our first goal was to be able to use the web for discussing specific problems, comparing solutions to exercises or building them online, monitoring students, helping in collecting and evaluating individual and/or group assignments, i.e. for enhancing the ordinary class offer.

After trying some other CMSs, the open-source software Moodle was chosen, for reasons briefly discussed in the next section. As in most CMSs, contents in Moodle are organized by courses in a tree structure, where each course corresponds to a subtree. Such hierarchical organization is convenient in the typical case of a student who wants to recover the content of a lecture he has missed, or the assignments due in a specific course, etc., but in many other cases the fact that the e-learning repository may only be browsed via a hierarchical traversal is a frustrating limitation.

Asynchronous availability, though generally considered among the most valuable peculiarities of web-based learning, is often mistaken with mere web publishing and is not yet well supported by suitable retrieval functions. This means that contents, questions and answers, possible remarks, etc., are available to users ... only if they can find them when they need them. The more students and teachers appreciate an elearning environment, the more its size tends to grow: good organization and powerful search mechanisms then become mandatory if users must be able to make full use of the large document base, not limited to the ability to retrieve a particular item whose existence is known in advance.

In order to improve the availability of resources to learners, a search mechanism shall be introduced that is orthogonal to the tree organization of courses, so that, for example, a student can compare the data structures and algorithms used in an introductory course on Algorithms with the analogous ones used in a Data Base course. Such parallel readings are a must in 
university studies: they should be recommended to students, and implicitly suggested, if not directly enforced, by the natural operation of the e-learning system. A CMS shall therefore support this kind of search, with results in forms easily readable and usable also by inexperienced learners.

An effort in this direction is the tool described here which couples Moodle with the dynamic taxonomies introduced by G. M. Sacco [2] and already applied in different contexts, as reported in the literature $[3,4,5$, $6,7]$. The tool will allow experimenting with dynamic taxonomies as a retrieval component in a CMS, where they are expected to play the original active function of prompting orthogonal visits of available resources by exploiting associations the user would not have thought of (and which are the specific contribution of dynamic taxonomies).

In the next section the Moodle system is briefly described, while in section 3 the taxonomy currently used in our prototype is introduced, with an example of search; screenshots of the user interface during the search are also shown. The prototype architecture is summarized in section 4, and finally some short conclusions are drawn in section 5 .

\section{Moodle}

Under the label of "electronically supported learning" (e-learning) we find different kinds of applications and underlying conceptions. In the nineties Dougiamas, when starting to develop Moodle, chose to build a web tool to support cooperative learning [8]. The results now suggest that Moodle is relatively successful as a tool to produce structured content with workbook-like answers; also in the view of its original designer, however, it is rather deficient in functionalities that allow to produce and manage an educational discourse using Moodle content-based framework [9]. Our work is in this direction.

Moodle is an open-source CMS implemented in PHP and relying on a MySQL RDBMS. The Department of Computer Science of the University of Turin chose Moodle among other CMSs because the open source was a promise of easier integration with the account system used for students and faculties and with a number of other locally developed services. In addition, the Università del Piemonte Orientale, located near Turin, was at that time a pioneer Moodle user with a worldwide distance course on Medical Emergency (http://Moodle.unipmn.it/em/); it still is a development center for Moodle.

The contents of a Moodle repository may have different formats, partitioned into two classes: one-way channels from teachers to learners, called resources, and bidirectional channels called activities. Resources typically are downloadable files, links, broadcast announcements, etc. Activities may be assignments, chats, forums, wikis, quizzes, lectures (organized in a graph structure whose traversal depends on the answers given by the reader to the questions proposed at each step). As concerns the retrieval of a particular piece of information, the current Moodle release is endowed with only a search engine in forum messages which, though useful, is of course insufficient for our intended purposes, and needs therefore to be replaced or paired with a more general mechanism. In addition, owing to its hierarchical structure common to many CMSs, Moodle rigidly separates the contents of different courses, thus making impossible the visit in an orthogonal way of the whole repository. This was consistent with the original project's goal of supporting cooperation within each single course community [8], but it was eventually found to be a too strong limitation in a cooperative environment where individual learning and teaching, though articulated in separate courses and subjects, may typically gain remarkable benefits from interaction and cooperation among all the members of the local community.

We thus decided to apply to our Moodle system the model of dynamic taxonomies, in order to make available an efficient search mechanism orthogonal both to courses and to elements formats: search may span over all kind of contributions (forums, lectures, assignments, etc.), though preserving each user's permissions and visibility.

\section{The dynamic taxonomy}

The taxonomy used to classify documents contains syntactic and semantic properties. Syntactic properties are automatically derived from the Moodle database; semantic properties are manually specified by positioning the document in one or, usually, several nodes of the dynamic taxonomy.

\subsection{The classification}

The highest levels of the dynamic taxonomy used in our prototype are shown in Figure 1, top left corner, and contain the following concepts:

a. Document Type (TIPO): for specifying each document's format, i.e., whether it is a .pdf, .ppt, etc. or a format specific to Moodle;

b. Faculty/Author (DOCENTE/AUTORE): name(s) of the document author(s);

c. Course (CORSO): the particular course where the document belongs;

d. Date (DATA): document insertion date and last modification date; 
e. Subject (ARGOMENTO): under this concept we have the topics from the ACM Computing Reviews Classification System taxonomy [10].

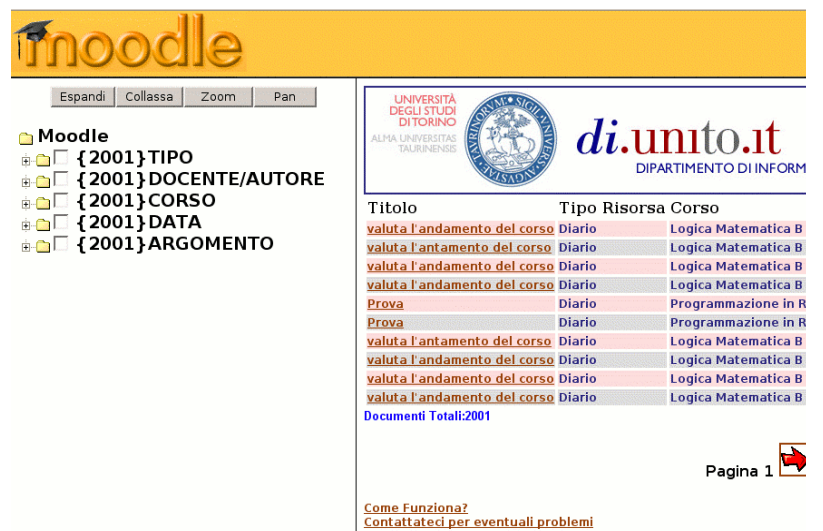

Figure 1. Highest levels of our dynamic taxonomy on top-left corner.

Concepts from a. to $\mathrm{d}$. represent the document "syntactical" properties automatically derived from the metadata in the Moodle database. As for the Subject classification, the ACM taxonomy has been chosen in view of a possible integration of the online CMS documents with our paper documents in the department library, i.e. books and journals. Subject classification is done by hand and in the currently used prototype is not yet synchronized with the document insertion in Moodle because we have chosen, as a first step, a lazyintegration between our prototype and the Moodle CMS. As often pointed out, updating the taxonomy with new concepts has no impact on the already classified contents $[3,4]$.

\subsection{An active function during retrieval}

When starting her/his search, a user is shown the prototype home page, in Figure 1, where the highest levels of the taxonomy, described in section 3.1, are presented on the top-left corner. Counters in Figure 1 tell the user that 2001 documents are classified in the taxonomy: all of them have automatically received a classification under syntactical concepts (TIPO, DOCENTE/AUTORE, CORSO, DATA). In this case all 2001 documents have also been classified under ARGOMENTO (subject). Indeed, during a different search, after other documents have been added to the considered content repository, the user may find that some documents have not yet been related to any node of the subtree having root ARGOMENTO in our dynamic taxonomy. In such a case, the counter number for ARGOMENTO will be smaller than the other counters related to syntactic properties. Above the taxonomy, in Figure 1, four buttons are available to execute the Zoom and Pan basic functions of a dynamic taxonomy system and to Expand or Collapse the whole tree.

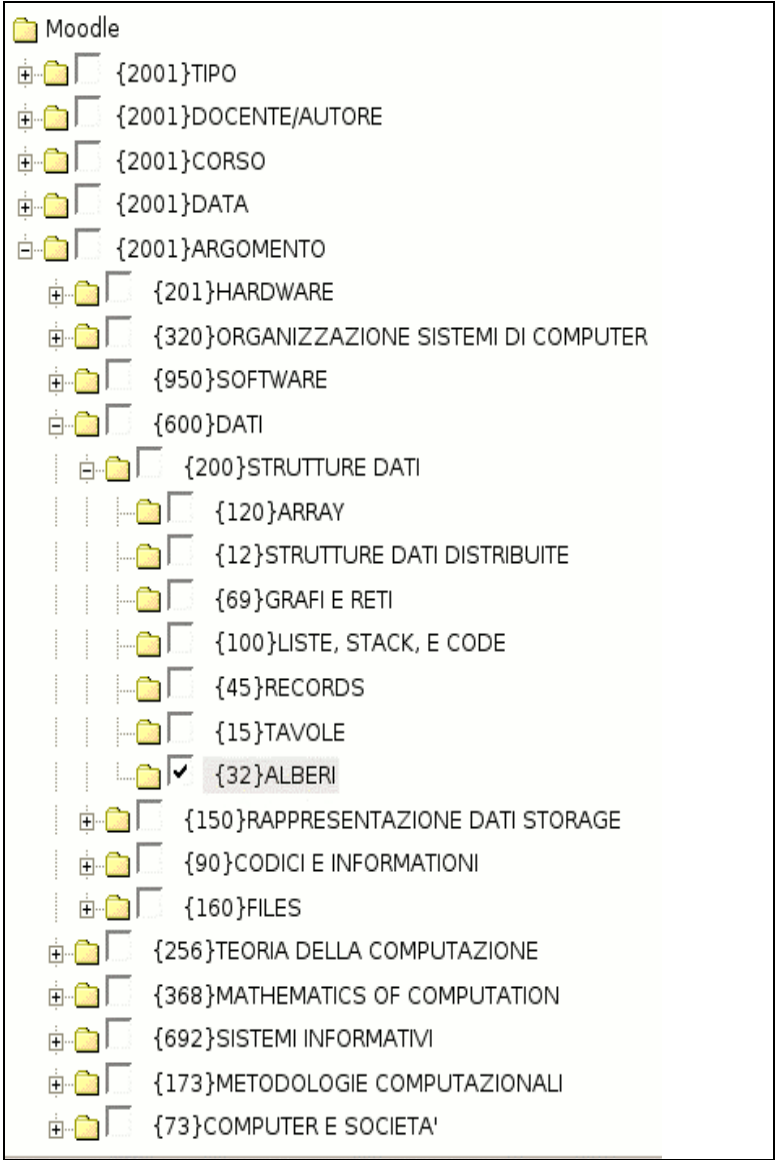

Figure 2. Expansion to the concept ALBERI (trees).

In Figure 2, below ARGOMENTO (Subject) and DATI (Data), the concept STRUTTURE DATI (Data Structures) was expanded down to the concept ALBERI (trees): the system found that 32 documents are classified under this concept, as shown by the counter. At this point the user of a conventional taxonomy would inspect these 32 documents (since tree is a terminal concept no further specializable). Instead, by using a dynamic taxonomy the user can zoom on his current set of interest, tree, and obtain the reduced taxonomy that indicates how the 32 documents are classified under other nodes of the dynamic taxonomy. In a reduced taxonomy the structure is the same as in the complete taxonomy, but specialized (or reduced) to concepts under which any of the documents classified, in this case, under ALBERI (trees) is also classified. Figure 3 partially shows the prototype window with the reduced taxonomy where the user sees that 24 documents out of 32 are from the course ALGORITMI E SPERIMENTAZIONI (Algorithms and Lab), 1 is in the 
RETI DI ELABORATORI (Computer nets) course, 1 in the BASI DI DATI (databases), etc. The last document found under the Computer nets course is presumably the one also classified under the concept ARGOMENTO (Subject) along the path ORGANIZZAZIONE SISTEMI DI COMPUTER (Computer Systems Organization), NETWORK, PROTOCOLl DI RETE (Net Protocols), APPLICATIVI (Applications).

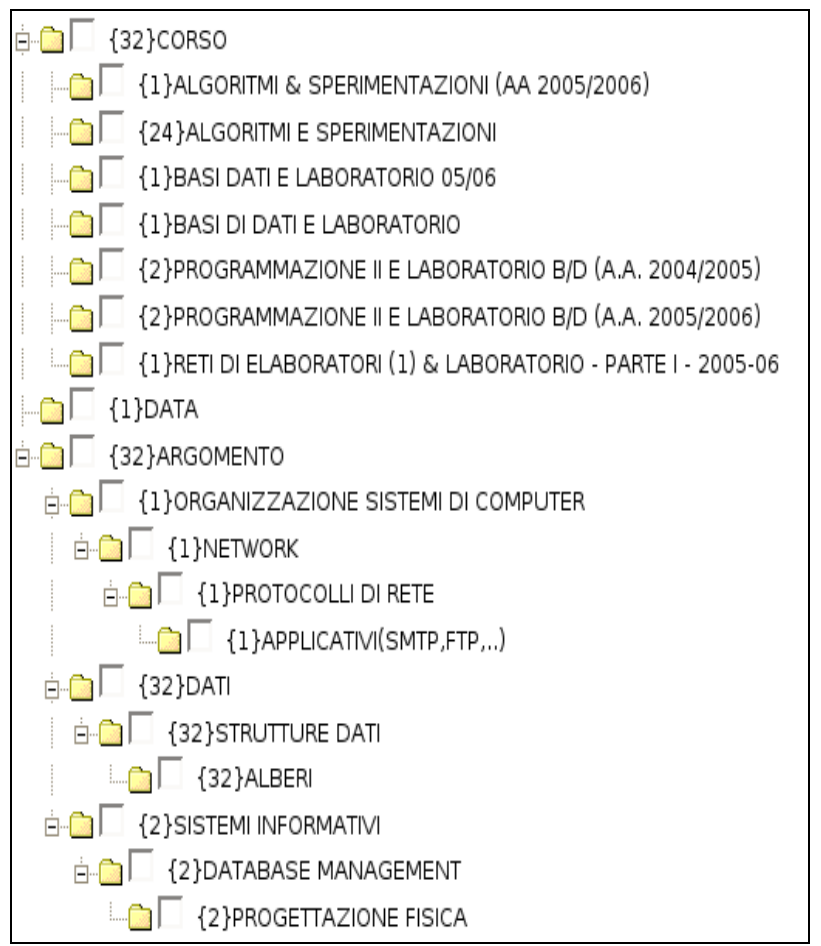

Figure 3. Reduced taxonomy wrt concept ALBERI.

In the part of the window not shown in Figure 3, the user can also find out that, for example, 8 out of 32 documents are .ppt documents, 5 are .pdf, 4 are Moodle quizzes, and 15 are forum messages.

The above example illustrates what we call the active function of the dynamic taxonomy model during retrieval. Given a concept in a node of the taxonomy, in our example tree in Figure 2, we find not only the documents classified under the topic tree reached through the path DATI - STRUTTURE DATI, but also the other concepts under which these 32 documents have been classified: for example the concepts ORGANIZZAZIONE SISTEMI DI COMPUTER (Computer Systems Organization), PROTOCOLLI DI RETE (Net Protocols), APPLICATIVI (Applications). The user finds both the documents he is looking for and the reduced taxonomy which describes the 32 documents, classified under "tree", in the same way as the initial taxonomy describes the entire document base [4]. The reduced taxonomic tree constitutes the current set of interest and generally prunes some concepts of the complete taxonomy under which none of the documents classified under "tree" is classified. Thus the user finds possibly interesting documents w. r. t. the search key, but in addition he is presented a summary of related concepts which have been used to classify at least one document also associated to the search key.

Since the extension of the taxonomy is used for inference, a lecturer can use the system to quickly find, for example, the courses where her students have been exposed to a given subject and thus get an idea of which knowledge they should already possess. Similarly a learner, while studying a subject from the perspective of a course $A$, can be helped by first considering it from the perspective of a course B he likes better, once he discovers that both $\mathrm{A}$ and $\mathrm{B}$ deal the given subject.

\section{Prototype architecture}

To manage the dynamic taxonomy our prototype uses Knowledge Processors' Universal Knowledge Processor (UKP), a commercial system chosen because of its extremely fast operations even on very large databases [11], as follows from the performance analysis for UKP in [12]. The prototype architecture has three basic subsystems.

MoodleSearch is the front-end web application, implemented using ASP, for document search; a screenshot of MoodleSearch home page is shown in Figure 1 where our taxonomy's first levels are displayed to the user on starting a new search. UKP primitives' calls are obviously issued by this component.

MoodleLoader, implemented in Java plus ASP scripts, loads Moodle documents identification codes (the URLs) and performs document syntactical classifications, i.e. by author, format, course (the course the document belongs to in the Moodle repository), dates of insertion and of last update.

MoodleClass, again written in Java plus ASP scripts, allows completing the document classification under the non-syntactically-derivable concepts: in our case under the ACM taxonomy. This classification is done by relating the document to a node of the subtree having root ARGOMENTO in the dynamic taxonomy.

The prototype has two kinds of uses. One is for completing the ACM classification and is reserved to course teachers. The second one is for retrieval purposes and is open to all Moodle members though preserving same user's permissions and visibility as in the Moodle system.

Documents not yet classified under the ACM classification concepts are found using concepts from 
the syntactical classification only. In our environment this is not a big limitation because many of our courses are named from ACM classification concepts.

\section{Conclusive remarks}

In Moodle the only search mechanism concerns forum messages within each single course. By using dynamic taxonomies as a retrieval system for Moodle documents, our goal is to have a search function extended to all courses and to all contents, i.e. to all activities and resources in Moodle. We consider this extension necessary as the content bases become larger, and easily very large, with the successful use of the CMS.

As for the prototype described here, several directions of work are open: first of all, an extensive use is needed for experimenting with different interfaces so as to find those most suitable for exploiting the active role of dynamic taxonomies integrated in a CMS. More specifically, we plan to design interfaces where the orthogonal visits of available resources, presented in a reduced taxonomy, are shown to the user during a search in some depth, thus making more attractive the exploration of different views and different teaching styles for a same subject.

\section{References}

[1] Courtiat, J.P., C. Davarakis, and T. Villemur (eds.), "Technology enhanced learning", Proceedings IFIP TC3 Workshop Springer, Toulouse, 2004

[2] G.M. Sacco, "Navigating the CD-ROM", Proceedings. Int'l Conf. Business of CD-ROM, Rome, 1987

[3] G.M. Sacco, "Dynamic taxonomies: a model for large information bases", IEEE Trans. on Knowledge and Data Engineering, 12, pp. 468-479, 2000

[4] G.M. Sacco, "Dynamic Taxonomies and guided Searches", J. of the American Society for Information Science and Technology, 57:6, pp. 792-797, April 200

[5] D. Wollersheim and W. Rahayu, "Methodology for creating a Sample Subset of Dynamic Taxonomy to Use in Navigating Medical Text databases", Proceedings IDEAS 2002 Conf., Edmonton, Canada, 2002

[6] Ping Yee, Kirsten Swearingen, Kevin Li, and Marti Hearst, "Faceted Metadata for Image Search and Browsing", Proceedings ACM CHI 2003 Conf., Fort Lauderdale, 2003

[7] E. Hyvönen, E. Mäkelä, M. Salminen, A. Valo, K.Viljanen, S. Saarela, M. Junnila and S. Kettula: "MuseumFinland - Finnish Museums on the Semantic Web", J. of Web Semantics, vol. 3, no. 2, pp. 25, 2005

[8] M. Dougiamas, "Developing tools to foster online educational dialogue", in K. Martin, N. Stanley and N. Davison (Eds), Teaching in the Disciplines/ Learning in Context, Proceedings 8th Annual Teaching Learning Forum, 119-123, Perth, 1998

[9] M. Dougiamas, "Improving the effectiveness of tools for Internet based education", in A. Herrmann and M.M. Kulski (Eds), Flexible Futures in Tertiary Teaching, Proceedings of the 9th Annual Teaching Learning Forum, Perth, 2000

[10] “Computing Reviews Classification System", ACM Computing Reviews, http://www.acm.org/class/1998/, 1998

[11] Knowledge Processors, Universal Knowledge Processor, 1999, www.knowledgeprocessors.com

[12] G.M. Sacco, "Efficient implementation of Dynamic Taxonomies", Tech Rep Univ. di Torino, Dip. di Informatica, 2003 